\title{
Have hybrid procedures replaced open aortic arch reconstruction in high-risk patients? A comparative study of elective open arch debranching with endovascular stent graft placement and conventional elective open total and distal aortic arch reconstruction
}

\author{
Rita Karianna Milewski, MD, PhD, Wilson Y. Szeto, MD, Alberto Pochettino, MD, \\ G. William Moser, CRNP, Patrick Moeller, BS, and Joseph E. Bavaria, MD
}

\begin{abstract}
Objective: Open total arch procedures have been associated with significant morbidity and mortality in patients with multiple comorbidities. Aortic arch debranching with endovascular graft placement, the hybrid arch procedure, has emerged as a surgical option in this patient population. This study evaluates the outcomes of a contemporary comparative series from one institution of open total arch and hybrid arch procedures for extensive aortic arch pathology.
\end{abstract}

\begin{abstract}
Methods: From July 2000 to March 2009, 1196 open arch procedures were performed, including 45 elective and 7 emergency open total arch procedures. From 2005 to 2009, 64 hybrid arch procedures were performed: 37 emergency type A dissections and 27 elective open arch debranchings. Hemiarch procedures were excluded.

Results: The hybrid arch cohort was significantly older $(P=.008)$ and had greater predominance of atherosclerotic pathophysiology $(P<.001)$. The incidence of permanent cerebral neurologic deficit was similar at $4 \%$ (1/27) for the hybrid arch cohort and 9\% (4/45) for the open aortic arch cohort. In-hospital mortality was similar at $11 \%(3 / 27)$ for the hybrid arch cohort and 16\% (7/45) for the open aortic arch cohort. However, in the open arch group, there was a significant difference in mortality between patients aged less than 75 years at $9 \%(3 / 34)$ and patients aged more than 75 years at $36 \%(4 / 11)(P=.05)$.

Conclusions: Hybrid arch procedures provide a safe alternative to open repair. This study suggests the hybrid arch approach has a lower mortality for high-risk patients aged more than 75 years. This extends the indication for the hybrid arch approach in patients with complex aortic arch pathology previously considered prohibitively high risk for conventional open total arch repair. (J Thorac Cardiovasc Surg 2010;140:590-7)
\end{abstract}

Aortic arch replacement for extensive and complex arch pathology is technically challenging. Open total arch and total arch plus descending aortic procedures can be accomplished electively with complex circulatory management and adjunct cerebral protection. However, these procedures, especially when staged, have been associated with significant, sometimes prohibitive morbidity and mortality for those patients with multiple comorbidities. $^{1-6}$ Thoracic endovascular repair has become recognized as a treatment alternative for uncomplicated aortic pathology of the descending thoracic aorta with acceptable midterm morbidity and mortality, and endovascular repair recently has been advocated for complicated aortic pathology, including type A dissections. ${ }^{1,2,7-10}$

\footnotetext{
From the Division of Cardiothoracic Surgery, Hospital of the University of Pennsylvania, Philadelphia, Pa.

Disclosures: None.

Read at the Eighty-ninth Annual Meeting of The American Association for Thoracic Surgery, Boston, Massachusetts, May 9-13, 2009.

Received for publication May 13, 2009; revisions received Feb 1, 2010; accepted for publication Feb 20, 2010.

Address for reprints: Rita Karianna Milewski, MD, PhD, 6 Silverstein Pavilion, 3400 Spruce Street, Philadelphia, PA 19104-4283 (E-mail: milewskr@uphs.upenn.edu). 0022-5223/\$36.00

Copyright (c) 2010 by The American Association for Thoracic Surgery doi: $10.1016 /$ j.jtcvs. 2010.02 .055
}

Hybrid arch procedures that combine open brachiocephalic debranching with concomitant antegrade endovascular stent graft placement in the aortic arch in a single-stage procedure have emerged as a treatment option for complicated aortic arch pathology. ${ }^{1,7,9,10}$ This complex pathology requires precise management and often modification of landing zones (LZs) for endovascular placement. Studies of brachiocephalic bypass procedures have demonstrated the feasibility of these procedures with acceptable mortality and neurologic outcomes. ${ }^{1,7,9,10}$

Appropriate selection of patients is essential when considering an endovascular versus open aortic approach for complex aortic arch pathology. This study evaluates the outcomes of a contemporary comparative series from one institution of open total arch, total arch plus descending aorta, and hybrid surgical procedures for extensive aortic arch pathology.

\section{MATERIALS AND METHODS \\ Patient Characteristics \\ From July 2000 to March 2009, we performed 1196 open arch proce- dures, of which 699 were elective hemiarch, 52 were total arch (45 elective and 7 emergency), and 43 were arch and descending aorta (38 elective and 5 emergency) procedures. For patients with distal arch and proximal descending procedures treated via a left thoracotomy, the most common pathophysiology was chronic dissection (16 patients, $43.2 \%$ ). The patients}




\section{Abbreviations and Acronyms \\ $\mathrm{ACP}=$ antegrade cerebral perfusion \\ $\mathrm{COPD}=$ chronic obstructive pulmonary disease \\ $\mathrm{CPB}=$ cardiopulmonary bypass \\ DHCA $=$ deep hypothermia and circulatory arrest \\ $\mathrm{LZ}=$ landing zone \\ $\mathrm{RCP}=$ retrograde cerebral perfusion}

in this cohort are not included for comparative analysis because most of them are currently being treated with thoracic endovascular stent grafting at the Hospital of the University of Pennsylvania. This study excluded all hemiarch procedures and included only elective procedures.

\section{Open Total Arch Cohort}

From 2000 to 2009 , we performed 45 elective total arch procedures. The mean age of patients was $62.8 \pm 13.5$ years. The pathophysiology included atherosclerotic in 26 patients $(58 \%)$ and chronic dissection in 16 patients $(39 \%)$. Patients with atherosclerotic pathology had a mean maximum aortic diameter of $7.3 \pm 1.5 \mathrm{~cm}$ (range, $5-11 \mathrm{~cm}$ ), as measured from the outer diameter of the aneurysm at its largest cross-sectional dimension.

\section{Hybrid Arch Cohort}

From 2005 to 2009, we performed 66 hybrid open endovascular procedures via a sternotomy. Thirty-seven were emergency Debakey I dissections with a distal "stented elephant trunk." Twenty-seven were "classic" elective open brachiocephalic debranching procedures with endovascular stent graft placement (hybrid arch). Hybrid arch procedures included 17 patients undergoing brachiocephalic debranching with endovascular stent graft placement alone (type I), 2 patients with concomitant ascending arch replacement (type II), and 8 patients with concomitant ascending arch replacement and extended stent graft coverage for atherosclerotic aneurysmal involvement of the ascending, aortic arch, and descending aorta or "mega-aorta" pathologies (type III) (Figure 1). The mean age of patients was $71.4 \pm 9.1$ years (range, $52-84$ years). The mean maximum size of atherosclerotic aneurysm was $7.3 \pm 1.6 \mathrm{~cm}$ (range, $4.4-10 \mathrm{~cm}$ ). Preoperative patient characteristics are summarized in Table 1 .

\section{Preoperative Evaluation and Operative Technique}

All patients were evaluated with multi-slice computed tomography angiogram (M2S, West Lebanon, $\mathrm{NH}$ ). Three-dimensional imaging was obtained and used for preoperative evaluation and proper sizing of the endovascular stent graft devices deployed in the arch.

Hybrid arch procedures were performed in a Hybrid Endosuite equipped with a universal floor-mounted angiographic C-arm system.

Intraoperative transesophageal echocardiogram was performed on all patients. Neuromonitoring with continuous electroencephalogram was used in all patients. All hybrid procedures also used somatosensory evoked potentials monitoring.

Comparative statistical analysis between the hybrid and control groups was conducted using SPSS version 15.0 (SPSS Inc, Chicago, Ill). The Mann-Whitney test was used to assess differences between groups for numeric variables. Fisher's exact test was used to assess differences between groups for categoric values.

\section{Surgical Technique}

Open total arch. A median sternotomy was performed in all patients. Arterial cannulation was accomplished in the ascending aorta for all patients undergoing open repair.
Cardiopulmonary bypass (CPB) and deep hypothermia and circulatory arrest (DHCA) were used in all open arch cases. Once core hypothermia was reached, circulatory arrest was initiated with retrograde cerebral perfusion (RCP). RCP was usually performed for several minutes before selective antegrade cerebral perfusion (ACP) via balloon-tipped cannulae, as previously described. ${ }^{8}$ Antegrade systemic circulation and rewarming were initiated after completion of the distal anastomosis and de-airing of the aorta. The supra-aortic vessels were reimplanted using a 4-branched graft. ${ }^{8}$ The proximal takeoff of each arch vessel was detached, and end-to-end anastomosis of the branched graft to the arch vessels was performed from the left subclavian to the innominate artery with sequential clamping. De-airing maneuvers were performed for each anastomosis before release of the distal clamp.

Hybrid arch debranching. For type I saccular arch aneurysms, the classic debranching procedure was performed using a 4-branched Dacron graft anastomosed in an end-to-side fashion along the greater curve of the ascending aorta, just distal to the sinotubular junction, to allow deployment of the stent graft in the ascending aorta without compromise of the proximal inflow anastomosis. The type IA $(n=2)$ procedure was performed off bypass using a sidebiting clamp on the ascending aorta. If a limited amount of aorta existed between the sinotubular junction and the ascending aorta, full CPB with aortic crossclamping and cardiac arrest using standard cardioplegia was required (type IB, $\mathrm{n}=15$ ). For patients with type II and III aortic pathology, DHCA was used. A segment of aorta just distal to the sinotubular junction was identified for the proximal anastomosis and normalization of the sinotubular junction performed using the 4-branch aortic graft. After the patient achieved profound hypothermia and a flat electroencephalogram, RCP was begun, and the aortic arch was opened. RCP was performed for a brief period before selective ACP while the proximal aortic arch was prepared for the type II $(n=2)$ or type IIIA $(n=4)$ and type IIIB $(n=4)$ hybrid procedures. After complete dissection of the brachiocephalic vessels off the aortic arch, selective ACP via balloon-tipped cannulae was initiated, and the distal aortic arch anastomosis was completed. For all hybrid arch procedures, the distal end-to-end anastomosis of the branched grafts to the arch vessels was sequentially performed for the left subclavian, left common carotid, and innominate arteries. The proximal takeoff of each arch vessel was detached using a stapling device. De-airing maneuvers were performed for each anastomosis before release of the distal clamp. Stent grafts were deployed antegrade during partial CPB in 21 of 27 patients (type IB, II, IIIA). The Gore TAG endoprosthesis (WL Gore and Associates, Inc, Newark, DE) was used in 25 of 27 patients. One Talent device (Medtronic, Inc, Minneapolis, MN) and 1 Cook TX2 device (Cook Medical Inc, Bloomington, IN) was used in the remaining patients. Deployment of stent graft devices was performed under fluoroscopy, and completion angiography was performed in all cases. Deployment of the stent graft was successful in $100 \%$ of cases. The average endovascular coverage length was $27.2 \pm$ $8.6 \mathrm{~cm}$ for type I, $15 \mathrm{~cm}$ for type II, and $32.1 \pm 16.8 \mathrm{~cm}$ for type III.

\section{RESULTS}

Patients undergoing hybrid procedures were significantly older than patients undergoing open total arch repair (71.3 \pm 9.6 years vs $62.8 \pm 13.5$ years, $P=.008$ ). There was a significant difference in aortic pathology between the 2 groups. Ninety-three percent of patients undergoing a hybrid procedure had atherosclerotic pathology, whereas $58 \%$ of patients undergoing open total arch repair had atherosclerotic pathology $(P<.001)$. Saccular atherosclerotic pathology was more prevalent in patients undergoing hybrid procedures $(63 \%$ vs $18 \%$ in the open total arch group, $P<.001$ ) (Table 1 ). Concomitant procedures were performed in both groups as described in Table 2. 

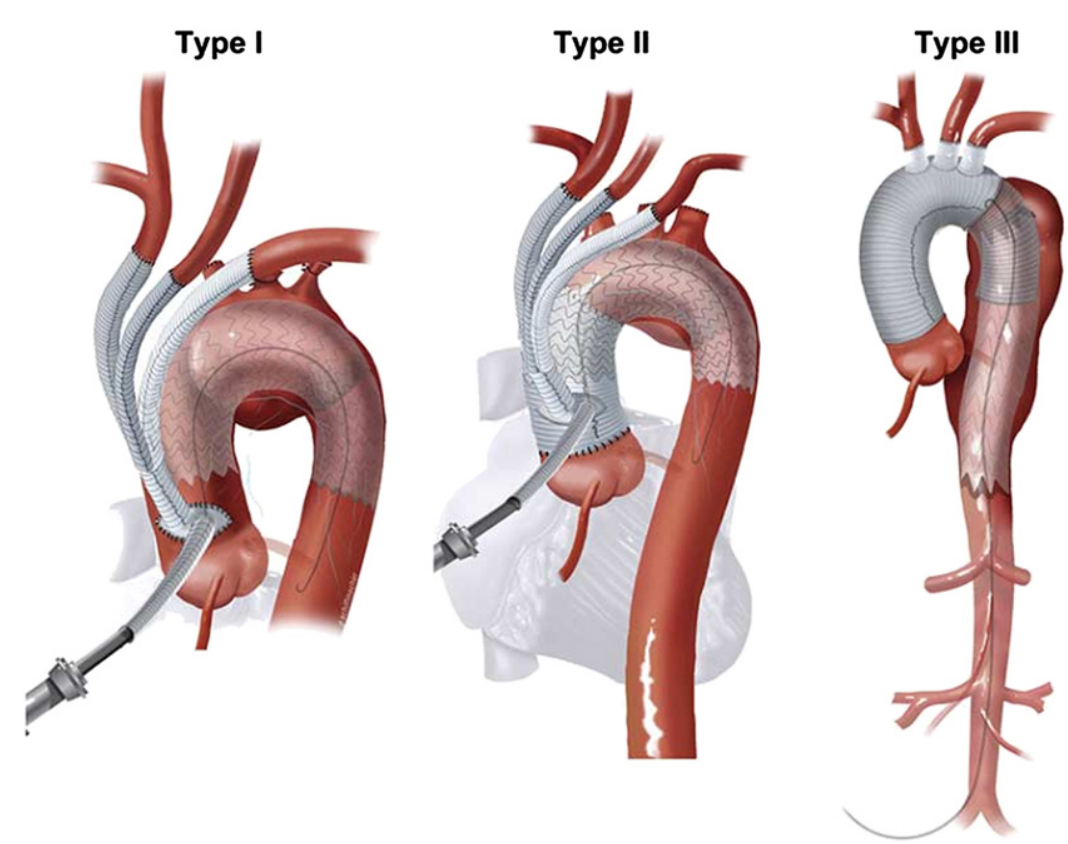

FIGURE 1. Type I aortic pathology: For type I saccular arch aneurysm, the "classic" debranching procedure can be performed to bypass the cerebral vessels. This procedure involves the end-to-side anastomoses of a 4-branched graft to the ascending aorta. This can be done off bypass using a sidebiting clamp on the ascending aorta (IA). If a limited amount of aorta exists between the STJ and the ascending aorta, a full CPB with crossclamp is required (IB). Type II aortic arch pathology: This aortic pathology has no adequate suitable native proximal aorta but has a distal aorta for antegrade deployment of the endovascular graft. However, this pathology requires reconstruction of LZ 0 with a 4-branch graft. Type III aortic arch pathology: For type III aortic arch pathology, or megaaorta, there is no adequate suitable native proximal ascending or descending aorta. The adequate suitable distal native aorta extends just proximal to the celiac artery. Mega-aorta also requires reconstruction of LZ 0 with a branched graft. The remaining mega-aorta is addressed by intraoperative antegrade deployment of the endovascular graft or by retrograde TEVAR during the same hospitalization. In some cases, an interval between the open debranching procedure and completion of aneurysmal exclusion with further endovascular stent grafting is required (IIIB).

Primary outcome data for overall in-hospital mortality were not significantly different between the hybrid $(11 \%)$ and open total arch repair $(16 \%)$ cohorts $(P=.739)$. The overall incidence of transient neurologic complications (cerebral and spinal cord) was not significantly different between cohorts. Transient cerebral neurologic deficit occurred in 5 patients $(11 \%)$ in the open total arch cohort and did not occur in patients undergoing hybrid arch procedures. Transient, reversible, spinal cord ischemia occurred in 3 patients $(11 \%)$ in the hybrid arch cohort and did not occur in patients in the total arch cohort. There was no significant difference in overall permanent neurologic complications between the hybrid $(13 \%)$ and open total arch $(9 \%)$ cohorts $(P=1.00)$. Permanent cerebral neurologic deficit, 2 embolic and 2 intracerebral hemorrhage secondary to anticoagulation therapy, occurred in 9\% (2 deaths) in the total arch group $(P=.644)$. A permanent cerebral neurologic deficit, embolic in origin, occurred in 1 patient $(4 \%$, survived) in the hybrid arch group. New postoperative paraplegia developed in 2 patients in the hybrid arch cohort $(7 \%)$, resulting in death before hospital discharge in both patients. The incidence of new postoperative renal insufficiency and new postoperative hemodialysis requirement was similar between the 2 cohorts (Table 3 ).
When the hybrid and open total arch cohorts are stratified by age, there are significant differences for in-hospital mortality between groups. For the open hybrid group, there is no significant difference between patients aged less than 75 years ( 2 deaths, $14 \%)$ and patients aged more than 75 years (1 death, $8 \%$ ). However, in the open total arch group, there is a significant difference in mortality between patients aged less than 75 years $(9 \%)$ and patients aged more than 75 years $(36 \%)(P=.05)($ Table 3$)$.

No endoleaks occurred in the hybrid arch group. Analysis of long-term, all-cause mortality revealed no significant difference in survival between groups (Figure 2). The mean length of follow-up was $13.3 \pm 2.6$ months in the hybrid arch cohort and $22.7 \pm 3.4$ months in the open total arch cohort.

\section{DISCUSSION}

The natural history of extensive pathologies of the aortic arch carries grave survival outcomes. Despite recent advances in operative techniques, aortic arch replacement for extensive and complex arch pathology remains a technical challenge. The conventional approach for these complex aortic repairs, direct open total arch or total arch plus descending aortic replacement, can be accomplished electively 
TABLE 1. Preoperative characteristics and comorbidities

\begin{tabular}{|c|c|c|c|c|c|}
\hline \multirow[b]{2}{*}{$\mathbf{N}$} & \multicolumn{2}{|c|}{ Hybrid } & \multicolumn{2}{|c|}{ Open total arch } & \multirow[b]{2}{*}{$P$ value } \\
\hline & & 27 & & 45 & \\
\hline Age, y & 71.3 & \pm 9.6 & 62.8 & \pm 13.5 & .008 \\
\hline Gender ( $\mathrm{N}$ male) & 16 & $62 \%$ & 24 & $52 \%$ & .63 \\
\hline Prior CVA & 3 & $12 \%$ & 6 & $13 \%$ & 1.00 \\
\hline Prior MI & 5 & $19 \%$ & 5 & $11 \%$ & .26 \\
\hline $\mathrm{CRF}$ & 3 & $12 \%$ & 2 & $4 \%$ & .344 \\
\hline Moderate/severe COPD & 12 & $44 \%$ & 5 & $11 \%$ & .003 \\
\hline Smoker & 18 & $67 \%$ & 23 & $51 \%$ & .077 \\
\hline \multicolumn{6}{|l|}{ Surgical history } \\
\hline Redo sternotomy & 6 & $22 \%$ & 15 & $33 \%$ & .424 \\
\hline CABG & 1 & $4 \%$ & 5 & $11 \%$ & .40 \\
\hline Root replace & 0 & & 4 & $9 \%$ & .29 \\
\hline AVR/root & 1 & $4 \%$ & 3 & $7 \%$ & 1.00 \\
\hline Asc aneurysm & 0 & $0 \%$ & 3 & $7 \%$ & .287 \\
\hline Type A dissection repair & 2 & $7 \%$ & 9 & $20 \%$ & .191 \\
\hline AAA & 5 & $19 \%$ & & & .006 \\
\hline \multicolumn{6}{|l|}{ Pathology } \\
\hline Atherosclerotic aneurysm & 26 & $96 \%$ & 26 & $58 \%$ & $<.001$ \\
\hline Fusiform & 9 & $33 \%$ & 18 & $40 \%$ & .623 \\
\hline Saccular & 17 & $63 \%$ & 8 & $18 \%$ & $<.001$ \\
\hline $\begin{array}{l}\text { High-grade/mobile } \\
\text { atheroma }\end{array}$ & 13 & $48.1 \%$ & 3 & $6.7 \%$ & $<.001$ \\
\hline Chronic dissection & 1 & $4 \%$ & 16 & $36 \%$ & $<.001$ \\
\hline Prior trauma & & & 1 & $2 \%$ & \\
\hline Other & & & 2 & $4 \%$ & \\
\hline
\end{tabular}

$A V R$, Aortic valve replacement; $A A A$, abdominal aortic aneurysm; $M I$, myocardial infarction; $C A B G$, coronary artery bypass grafting; $C V A$, cerebral vascular accident (a documented history of stroke with loss of neurologic function with residual symptoms at least $72 \mathrm{~h}$ after onset); $C R F$, chronic renal failure (a documented history of renal failure or a creatinine $>2.0$ ); COPD, chronic obstructive pulmonary disease (moderate, forced expiratory volume in $1 \mathrm{sec} 50 \%-59 \%$ of predicted or receiving chronic steroid therapy aimed at lung disease; severe, forced expiratory volume in $1 \mathrm{sec}<50 \%$ of predicted or room air $\mathrm{Po}_{2}<60$, or $\mathrm{PCO}_{2}>50$. A smoker is one with a history of any form of tobacco use.

with sophisticated circulatory management and adjunct cerebral protection. However, these procedures, especially when staged, have been associated with significant, sometimes prohibitive, morbidity and mortality for patients with multiple comorbidities. ${ }^{1-6}$

Treatment of extensive aortic aneurysms requires innovative surgical techniques and sophisticated patient selection. Operative approaches for complex aortic pathologies have undergone an evolution during the past 25 years; however, the optimal surgical technique has not been definitively established. The results of recent studies demonstrate that open aortic arch replacement can be undertaken under elective circumstances using contemporary techniques with acceptable operative mortality and morbidity. ${ }^{6,11,12}$

This study analyzed a contemporary cohort of patients who underwent elective open total aortic arch procedures using a branched graft, end-to-end arch vessel anastomotic technique. These open total aortic arch procedures all used a single standardized ACP and systemic circulatory protocol throughout the time frame of the study. Although it is diffi- cult to compare results from multiple institutions, our outcomes are consistent with those of other series of open aortic arch procedures. ${ }^{2-6,11,12}$ In our study, the results of open aortic procedures revealed an overall mortality of $16 \%$ and a permanent cerebral neurologic deficit of $9 \%$. Other contemporary series report similar incidences of mortality and permanent cerebral neurologic deficit. ${ }^{3-5,13}$

Although the results of open total arch repair have improved, the morbidity and mortality associated with conventional repair using CPB and DHCA are not insignificant. For complicated aortic arch pathology in patients with multiple prohibitive comorbidities, the hybrid procedure can be performed with acceptable morbidity and mortality. 1,7,9,10 This complex aortic pathology requires precise management and often modification of LZs for endovascular placement. ${ }^{1,7,10}$

In our series, for patients with type I saccular arch pathology $(\mathrm{n}=17$ ) with suitable native proximal and distal LZs, the classic debranching procedure using a 4-branched graft and antegrade deployment of the endovascular stent into the native ascending aorta was performed either off (type IA) or on (type IB) CPB. For type II arch pathology $(n=2)$ (unsuitable proximal LZ but suitable distal LZ), ascending aortic reconstruction of LZ 0 with the 4-branched graft and endovascular stent deployment into a Dacron ascending aorta was performed. For patients with type III "mega-aorta", pathology $(\mathrm{n}=8)$ (unsuitable proximal LZ and distal LZ), total arch replacement with simultaneous "frozen elephant trunk" endovascular stent deployment (type IIIA, $\mathrm{n}=4$ ) or early (generally $<2$ weeks) "stented elephant trunk" (type IIIB, $\mathrm{n}=4$ ) was performed. The types of hybrid procedures are illustrated in Figure 1.

Analysis of the 27 patients in the hybrid arch cohort revealed that $93 \%$ had atherosclerotic aneurysms. The overall mortality was $11 \%$ in this cohort of patients. This is consistent with mortality seen in other hybrid series $(0 \%-$ $15 \%){ }^{1,7,9,10,14}$ In our hybrid arch group, the incidence of permanent cerebral neurologic deficit was 4\%. Paraplegia occurred in 2 patients $(7 \%)$ and is consistent with other hybrid series, which report a $0 \%$ to $10 \%$ incidence of paraplegia. $1,7,9,10,14$ Both of these cases occurred early in our series. One patient had standard endograft deployment in the proximal descending aorta. The second patient had extensive aneurysmal disease, including abdominal aortic aneurysm requiring distal stent deployment to the celiac artery. This extensive endovascular stent placement in conjunction with abdominal aortic aneurysm has been associated with spinal cord injury. ${ }^{14}$ Shimamura and colleagues, ${ }^{15}$ in a series of 126 elective and emergency hybrid procedures, report actuarial survival estimates of $81.1 \%$, $63.3 \%$, and $53.7 \%$ at 1,5 , and 8 years after the procedure, respectively. In our series the cumulative survival for the hybrid procedure is $63 \%$ at 4 years (Figure 2).

A major advantage of the hybrid arch procedure is that mega-aorta pathology can be repaired in patients in 1 
TABLE 2. Concomitant procedures and operative times

\begin{tabular}{|c|c|c|c|c|c|c|c|c|c|c|}
\hline \multirow[b]{2}{*}{$\mathbf{N}$} & \multicolumn{2}{|c|}{ Hybrid } & \multicolumn{2}{|c|}{ Type 1 hybrid } & \multicolumn{2}{|c|}{ Type 2 hybrid } & \multicolumn{2}{|c|}{ Type 3 Hybrid } & \multicolumn{2}{|c|}{ Open total arch } \\
\hline & \multicolumn{2}{|c|}{27} & \multicolumn{2}{|c|}{17} & \multicolumn{2}{|c|}{2} & \multicolumn{2}{|c|}{8} & \multicolumn{2}{|c|}{45} \\
\hline Arch alone & 13 & $48 \%$ & 13 & $76 \%$ & & & & & 8 & $18 \%$ \\
\hline $\operatorname{Arch}+\mathrm{CABG}$ & 3 & $11 \%$ & 3 & $18 \%$ & & & & & 1 & $2 \%$ \\
\hline $\operatorname{Arch}+\mathrm{AVR}$ & 1 & $4 \%$ & 1 & $6 \%$ & & & & & 1 & $2 \%$ \\
\hline $\operatorname{Arch}+\operatorname{Asc}$ & 2 & $7 \%$ & & & 2 & $100 \%$ & & & 11 & $25 \%$ \\
\hline $\mathrm{Arch}+\mathrm{Asc}+\mathrm{CABG}$ & & & & & & & & & 6 & $13 \%$ \\
\hline $\operatorname{Arch}+\mathrm{Asc}+$ Root & & & & & & & & & 11 & $25 \%$ \\
\hline $\mathrm{Arch}+\mathrm{Asc}+\mathrm{Root}+\mathrm{CABG}$ & & & & & & & & & 3 & $6.7 \%$ \\
\hline Arch + Asc + Root/AVR + Desc(Mega $)$ & 1 & $4 \%$ & & & & & 1 & $13 \%$ & 3 & $6.7 \%$ \\
\hline Arch + Asc + Desc(Mega $)$ & 7 & $26 \%$ & & & & & 7 & $88 \%$ & 1 & $2 \%$ \\
\hline $\mathrm{CPB}$ time $(\mathrm{m})^{*}$ & 220.8 & \pm 61.5 & & & & & & & 282 & \pm 55.7 \\
\hline Crossclamp time $(\mathrm{m})^{*}$ & 83.2 & \pm 62.1 & & & & & & & 192.5 & \pm 65.1 \\
\hline
\end{tabular}

$C A B G$, Coronary artery bypass grafting; $A V R$, aortic valve replacement; $C P B$, cardiopulmonary bypass. ${ }^{*} P$ value between groups $<.001$.

operation, during the same hospitalization, or an interval generally less than 2 weeks. In patients with mega-aorta pathology receiving a 2-stage "elephant trunk" repair, mortality incidences of up to $9.5 \%$ for the first stage, $8 \%$ to $11 \%$ in the interval between stages, and $7.0 \%$ for the second stage have been reported. ${ }^{2,3}$ For patients who do not return after the first procedure, the incidence of mortality is reported as high as $32 \%$ to $35 \%{ }^{2,3}$ For extensive aortic arch pathologies, the results of a single-stage procedure using a thoracosternotomy procedure include mortality incidence of $6.6 \%$ to $14 \%$, permanent neurologic deficit of $0 \%$ to $20 \%$, and paraplegia in $1.4 \%$ to $14 \% .^{19,20}$ These results suggest that an alternative treatment is needed for patients with high morbidity due to extensive aortic pathology. A recent series reported an improved outcome for these

TABLE 3. Outcomes

\begin{tabular}{|c|c|c|c|c|c|}
\hline \multirow[b]{3}{*}{ In-hospital mortality } & \multirow{2}{*}{\multicolumn{2}{|c|}{$\begin{array}{l}\text { Hybrid } \\
N=27\end{array}$}} & \multirow{2}{*}{\multicolumn{2}{|c|}{$\begin{array}{c}\text { Open total arch } \\
\mathrm{N}=45 \\
\end{array}$}} & \multirow{3}{*}{$\frac{\boldsymbol{P}}{\frac{\boldsymbol{P}}{\text { value }}} \frac{\mathrm{.739}}{}$} \\
\hline & & & & & \\
\hline & 3 & $11 \%$ & 7 & $16 \%$ & \\
\hline Stroke & 1 & $4 \%$ & 4 & $9 \%$ & .644 \\
\hline Transient neurologic deficit & 0 & & 5 & $11 \%$ & .15 \\
\hline Permanent paraplegia & 2 & $7 \%$ & 0 & & .137 \\
\hline $\begin{array}{l}\text { Reversed spinal } \\
\text { cord ischemia }\end{array}$ & 3 & $11 \%$ & 0 & & .049 \\
\hline renal failure & 5 & $19 \%$ & 5 & $11 \%$ & .486 \\
\hline $\begin{array}{l}\text { Renal failure } \\
\text { requiring new hemodialysis }\end{array}$ & 3 & $11 \%$ & 3 & $7 \%$ & .665 \\
\hline Reoperation for bleeding & 0 & & 1 & $2 \%$ & 1.00 \\
\hline Afibrillation & 9 & $33 \%$ & 13 & $29 \%$ & .793 \\
\hline $\begin{array}{l}\text { Mean hospital } \\
\text { stay (d) }\end{array}$ & 20.1 & 15.9 & 17.5 & 16.2 & \\
\hline
\end{tabular}

Age stratification and mortality

\begin{tabular}{clrlr}
\hline & \multicolumn{4}{c}{ In-hospital mortality } \\
\cline { 2 - 5 } & \multicolumn{3}{c}{ Hybrid arch } & Open total arch \\
\hline$<75 \mathrm{y}$ & 2 & $14 \%$ & 3 & $9 \%$ \\
$>75 \mathrm{y}$ & 1 & $8 \%$ & 4 & $36 \%$ \\
\hline
\end{tabular}

patients using a "frozen" elephant trunk repair. ${ }^{21} \mathrm{We}$ have essentially abolished the use of an extended interval "staged elephant trunk" procedure at the Hospital of the University of Pennsylvania. In our series, patients undergoing staged repair of their mega-aorta in a conventional manner (without hybrid arch and endovascular procedure) had a mortality of $40 \%$. Alternatively, when incorporating a hybrid arch repair, and descending aortic endovascular placement, the incidence of mortality was reduced to $11 \%$.

The hybrid approach avoids a left thoracotomy by using a stent graft as an alternative to an open distal anastomosis. Although the incidence of mortality for patients undergoing open distal/descending repair (excluding thoracoabdominal aneurysm repair) was only $3 \%(1 / 38)$ at the Hospital of the University of Pennsylvania, these patients were 18 years younger on average than patients undergoing hybrid procedures $(53.0$ vs 71.3 years $)(P<.001)$. The incidence of pulmonary complications in patients undergoing descending aneurysm repair has been reported as up to $28 \%{ }^{16-18}$ Of open distal cases at the Hospital of the University of Pennsylvania, $11 \%$ required lung decortication. This is especially important because $67 \%(18 / 27)$ of the hybrid cohort compared with $51 \%$ (23/45) of the open total arch cohort $(P=.077)$ had a significant smoking history. For the hybrid arch population, $44 \%$ (12/27) had moderate-tosevere chronic obstructive pulmonary disease (COPD) compared with $11 \%(5 / 45)$ of the open $\operatorname{arch}$ group $(P=.003)$.

Preoperative renal failure has been reported as a predictor of mortality in aortic arch replacement. ${ }^{11}$ In our series, chronic renal failure was present preoperatively in $12 \%$ of the open hybrid cohort compared with $4 \%$ of the open total $\operatorname{arch}$ cohort $(P=.344)$. Although the majority of our hybrid procedures were performed on CPB (25/27), the hybrid procedure avoided DHCA, including potential renal ischemia in $63 \%$ of patients. Overall postoperative renal failure was similar in both cohorts (Table 3).

To determine an algorithmic approach for patient selection for either hybrid or open arch repair, criteria must be 


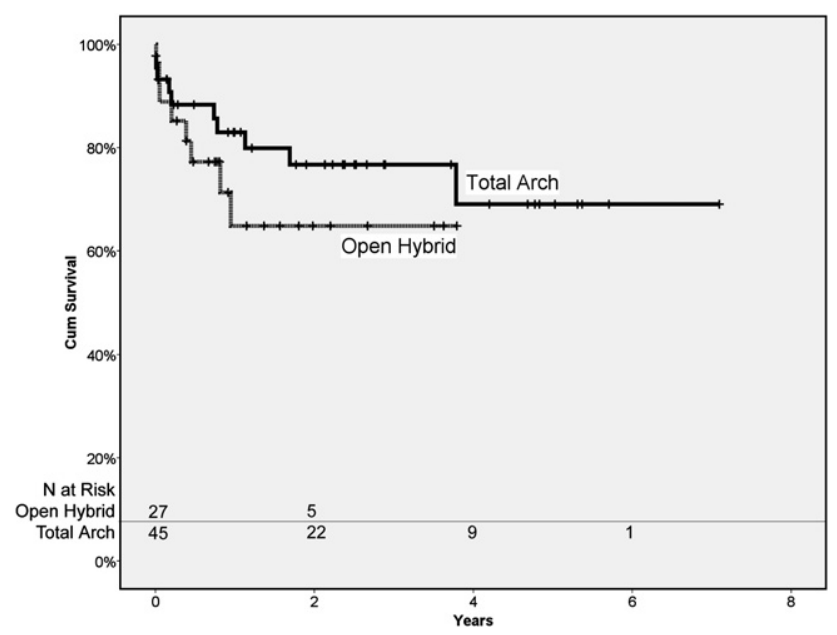

FIGURE 2. Kaplan-Meier survival plot, all causes of death. $P$ value between groups is .32 (log rank).

based on presenting comorbidities, such as a significant smoking history, moderate-to-severe COPD, saccular aneurysm with significant atherosclerotic burden, and high-grade or mobile atheroma. As older populations with additional comorbidities present with aortic pathologies, innovative methods of treatment must be developed to achieve acceptable outcomes.

In addition to age and preoperative comorbidities, patient selection for operations on the aortic arch must parallel the pathophysiology of the aorta. Reports have shown atherosclerotic pathology is a predictor of perioperative stroke. ${ }^{4,25}$ In our series, patients selected for hybrid procedure were older by 10 years $(P=.008)$ and $93 \%$ had an atherosclerotic aortic pathology with an extensive aortic arch and brachiocephalic atherosclerotic burden, compared with only $45 \%$ of patients with atherosclerotic aneurysms in those undergoing open total $\operatorname{arch}(P<.001)$. Delineation of atherosclerotic aneurysms in patients undergoing hybrid procedures revealed that $63 \%(17 / 27)$ were saccular arch aneurysms compared with $18 \%(8 / 45)$ in patients undergoing open total arch procedures $(P<.001)$. Of the fusiform aneurysms in patients undergoing hybrid procedures, $88 \%$ (8/9) were mega-aorta compared with $28 \%(5 / 18)$ in patients undergoing open total arch procedures. Previously, these patients were considered prohibitively high risk for conventional open arch repair because of the associated high stroke risk. Recent series of total arch repair for large atherosclerotic arch aneurysms still report in-hospital mortality ranging from $6.3 \%$ to $20 \%$ and incidence of stroke up to $12 \% .^{1,3,4,6,25}$ In our series, delineation of atherosclerotic burden revealed 48.1\% (13/ 27 ) of the hybrid arch cohort had high-grade or mobile atheroma compared with $6.7 \%(3 / 45)$ of the open arch cohort $(P<.001) .{ }^{25}$ On the basis of this high atherosclerotic burden, we perform end-to-end arch vessel anastomosis to ensure removal of proximal atherosclerotic disease and decrease the risk of embolic stroke. In our series, the overall incidence of permanent cerebral neurologic deficit was $4 \%$ in patients undergoing hybrid arch procedures and $9 \%$ for patients undergoing open total arch procedures $(P=.644)$. Stratified by age, the incidence of permanent cerebral neurologic deficit for patients undergoing hybrid arch procedures was $4 \%$ (1/14) for those aged less than 75 years and $0 \%$ for those aged more than 75 years. For patients undergoing open total arch procedures, the incidence of permanent cerebral neurologic deficit was $8.8 \%(3 / 34)$ for those aged less than 75 years and $9.1 \%(1 / 11)$ for those aged more than 75 years.

The results of our series and others suggest that age is a univariate predictor of mortality. ${ }^{5,22}$ Recent studies on open ascending and aortic arch repairs have revealed that mortality is $7.4 \%$ for patients aged more than 75 years and $13.5 \%$ for patients aged more than 80 years, with a stroke incidence of $3.7 \%$ and $8 \%$, respectively. ${ }^{23,24}$ However, none of these patients underwent an open total arch repair. In our series, for patients aged more than 75 years, inhospital mortality for hybrid arch cases was $8.3 \%(1 / 12)$ and $36 \%(4 / 11)$ in open total arch repair cases $(P=.049)$.

Patient selection indications for open total aortic arch procedures include our exclusion criteria for hybrid arch reconstruction, that is, younger patients, patients with infection, and patients with collagen vascular disease, such as Marfan syndrome. For patients undergoing open total arch repair, there was a tendency toward multiple complex concomitant cardiac procedures.

In developing algorithmic selection criteria for patients who would benefit from hybrid procedures, our criteria are based on age, aortic pathology, and comorbidities. These include aneurysms proven by M2S as saccular or mega-aorta with significant atherosclerotic burden and transesophageal echocardiogram evidence of high-grade or mobile atheroma. These criteria also include patients with significant smoking history and moderate-to-severe COPD who may not tolerate a left thoracotomy or a thoracosternotomy, patients with chronic renal failure, patients who would not tolerate an open staged procedure because of comorbidities, and patients with other significant preoperative neurologic compromise (previous cerebral vascular accident or paraplegia). By using these criteria, $71 \%$ (4 patients aged $>75$ years and 1 patient aged $<75$ years) of the patients who died in the open aortic arch cohort would now be treated at the Hospital of the University of Pennsylvania with the hybrid operative technique.

\section{CONCLUSIONS}

Analysis of overall mortality and morbidity of both hybrid and open total arch procedures demonstrates similar outcomes at the Hospital of the University of Pennsylvania. For patients with multiple comorbidities, including advanced age, large atherosclerotic burden, and end-organ compromise, the hybrid procedure allows for exclusion of 
the aneurysm while preserving renal, lung, and cerebral function with outcomes comparable to the open procedure.

Our results indicate that hybrid arch procedures have their primary benefit in high-risk cases, especially elderly patients aged more than 75 years with complex aortic arch pathology, such as large saccular aneurysms or mega-aorta, who were previously considered prohibitively high risk for conventional open total arch repair. Hybrid arch procedures provide a safe alternative to open repair and extend the indication of the approach to this high-risk cohort with midterm survival outcomes similar to those for the open total arch procedure. For younger patients with fewer comorbid risk factors and with exclusion criteria to hybrid arch repair, the open total arch procedure remains a reasonable option.

\section{References}

1. Szeto W, Bavaria JE, Bowen F, Woo E, Fairman R, Pochettino A. The hybrid total arch repair: brachiocephalic bypass and concomitant endovascular aortic arch stent graft placement. J Card Surg. 2007;22:97-102.

2. Svensson LG, Kim K, Blackstone E, Alster J, McCarthy P, Greenberg R, et al. Elephant trunk procedure: newer indications and uses. Ann Thorac Surg. 2004; 78:109-16.

3. Estrera A, Miller C, Porat E, Huynh T, Winnerkvist A, Safi HJ. Staged repair of extensive aortic aneurysms. Ann Thorac Surg. 2002;74:S1803-5.

4. Ergin MA, Galla J, Lansman S, Quintana C, Bodian C, Griepp R. Hypothermic circulatory arrest in operations on the thoracic aorta determinants of operative mortality and neurologic outcome. J Thorac Cardiovasc Surg. 1994;107:788-92.

5. Svensson LG, Crawford ES, Hess KR, Coselli JS, Raskin S, Shenaq SA, et al. Deep hypothermia with circulatory arrest. Determinants of stroke and early mortality in 656 patients. J Thorac Cardiovasc Surg. 1993;106:19-28.

6. Sundt TM, Orszulak TA, Cook DJ, Schaff HV. Improving results of open arch replacement. Ann Thorac Surg. 2008;86:787-96.

7. Hughes GC, Daneshmand MA, Swaminathan M, Nienaber JJ, Bush EL, Husain AH, et al. Real world" thoracic endografting: results with the Gore TAG device 2 years after U.S. FDA approval. Ann Thorac Surg. 2008;86:1530-8.

8. Pochettino A, Brinkman WT, Moeller P, Szeto WY, Moser GW, Cornelius K, et al. Proximal descending thoracic stent grafting via the open arch during standard repair for acute Debakey I aortic dissection prevents development of dissecting thoracoabdominal aortic aneurysms. Ann Thorac Surg. 2009;88:482-90.

9. Czerny M, Zimpfer D, Fleck T, Hofmann W, Schoder M, Cejna M, et al. Initial results after combined repair of aortic arch aneurysms by sequential transposition of the supra-aortic branches and consecutive endovascular stent-graft placement. Ann Thorac Surg. 2004;78:1256-60.

10. Bavaria JE, Appoo JJ, Makaroun MS, Verter J, Yu ZF, Mitchell RS, Gore TAG Investigators. Endovascular stent grafting versus open surgical repair of descending thoracic aortic aneurysms in low-risk patients: a multicenter comparative trial. J Thorac Cardiovasc Surg. 2007;133:369-77.

11. Kazui T, Yamashita K, Washiyama N, Terada H, Bashar AHM, Suzuki K, et al. Aortic arch replacement using selective cerebral perfusion. Ann Thorac Surg. 2007;83:S796-8.

12. Okita Y, Ando M, Minatoya K, Kitamura S, Takamoto S, Nakajima N. Predictive factors for mortality and cerebral complications in arteriosclerotic aneurysm of the aortic arch. Ann Thorac Surg. 1999;67:72-8.

13. Gega A, Rizzo JA, Johnson MH, Tranquilli M, Farkas EA, Elefteriades JA. Straight deep hypothermic arrest: experience in 394 patients supports its effectiveness as a sole means of brain preservation. Ann Thorac Surg. 2007;84: 759-67.

14. Flores J, Kunihara T, Shiiya N, Yoshimoto K, Matsuzaki K, Yasuda K. Extensive deployment of the stented elephant trunk is associated with an increased risk of spinal cord injury. J Thorac Cardiovasc Surg. 2006;131:336-42.

15. Shimamura K, Kuratani T, Matsumiya G, Kato M, Shirakawa Y, Takano H, et al. Long-term results of the open stent-grafting technique for extended aortic arch disease. J Thorac Cardiovasc Surg. 2008;135:1261-9.

16. Conrad MF, Crawford RS, Davison JK, Cambria RP. Thoracoabdominal aneurysm repair: a 20-year perspective. Ann Thorac Surg. 2007;83:S856-61.
17. Westaby S, Katsumata T. Proximal aortic perfusion for complex arch and descending aortic disease. J Thorac Cardiovasc Surg. 1998;115:162-7.

18. Svensson LG, Crawford ES, Hess KR, Coselli JS, Safi HJ. Variables predictive of outcome in 832 patients undergoing repairs of the descending thoracic aorta. Chest. 1993;104:1248-53.

19. Kouchoukos NT, Mauney MC, Masetti P, Castner CF. Optimization of aortic arch replacement with a one-stage approach. Ann Thorac Surg. 2007;83:S811-4.

20. Doss M, Woehleke T, Wood JP, Martens S, Greinecker GW, Moritz A. The clamshell approach for the treatment of extensive thoracic aortic disease. J Thorac Cardiovasc Surg. 2003;126:814-7.

21. Karck M, Kamiya H. Progress of the treatment for extended aortic aneurysms; is the frozen elephant trunk technique the next standard in the treatment of complex aortic disease including the arch? Eur J Cardiothorac Surg. 2008;33:1007-13.

22. Gutsche JT, Cheung AT, McGarvey ML, Moser WG, Szeto W, Carpenter JP, et al. Risk factors for perioperative stroke after thoracic endovascular aortic repair. Ann Thorac Surg. 2007;84:1195-200.

23. Ergin MA, Galla JD, Lansman SL, Quintana C, Bodian C, Griepp RB. Hypothermic circulatory arrest in operations on the thoracic aorta: determinants of operative mortality and neurologic outcome. J Thorac Cardiovasc Surg. 1994; 107:788-92.

24. Czerny M, Fleck T, Zimpfer D, Dworschak M, Hofmann W, Hutschala D, et al. Risk factors of mortality and permanent neurologic injury in patients undergoing ascending aortic and arch repair. J Thorac Cardiovasc Surg. 2003;126: 1296-301.

25. Pallav J, Shah JP, Estrera AL, Miller CC, Lee TY, Irani AD, et al. Analysis of ascending and transverse aortic arch repair in octogenarians. Ann Thorac Surg. 2008;86:774-9

\section{Discussion}

Dr Yutaka Okita (Kobe, Japan). I have no disclosures. Drs Milewski and Bavaria's group are to be congratulated on reporting a systematic approach to aortic arch pathologies. They compared 27 patients who underwent elective open hybrid arch procedures and 45 patients who underwent elective open total arch replacement at the University of Pennsylvania. Although patients' backgrounds were not similar and the number of patients was small, they concluded the hybrid arch procedures provided better outcome in elderly patients.

In 1996, Kato introduced an open hybrid stent-graft approach to the aortic arch aneurysm, and he also applied the off-pump debranching endograft method in 1999. According to the annual survey performed by the Japanese Association for Thoracic Surgery, from 2005 to 2007, 11,000 elective arch procedures were performed in Japan. A total of 5700 patients (51\%) had total arch replacement with a hospital mortality of $7.1 \%$, and 465 patients (4\%) had hybrid stent-grafting with a hospital mortality of $6.9 \%$. So both procedures, open total arch replacement or hybrid procedures, had similar results regarding hospital mortality in Japan.

Our own results in surgical open repair of the aortic arch in Kobe University are as follows. From March 2002 to December 2008, we performed 231 total arch replacements using ACP at a tympanic temperature of $25^{\circ} \mathrm{C}$. There were 166 elective cases, including 44 chronic aortic dissections. The mean age was 70 years. We found 6 hospital deaths (3.6\%) and 9 new strokes (5.4\%). For 88 patients aged more than 75 years ( $53 \%$ of our patients), hospital mortality was $4.5 \%$ (4) and stroke incidence was $5.7 \%$ (5). So by using sophisticated techniques of brain protection and sealed branch grafts, clinical outcome was similar, even in elderly patients. I have several questions for Dr Milewski.

In the hybrid cases, you do not need longer periods of circulatory arrest time under deep hypothermia; however, I found a relatively higher incidence of postoperative paraplegia or paraparesis. What 
were the causes of these complications and how do you prevent them?

Dr Milewski. There were 2 cases of postoperative paraplegia in our hybrid group. The first case had normal deployment of the antegrade graft. The second case occurred in a patient who had undergone an abdominal aortic aneurysm replacement and had a pavement of the stent-graft for mega-aorta all the way down to the celiac access, which has been shown to be high risk for paraplegia or spinal cord ischemia. Both of these cases occurred early in our series, before June of 2006. Since that time, we haven't had any. Part of this could be anatomy alone, and obviously part of it is the learning curve, not only in the actual deployment but also in the learning curve in patient selection.

Dr Okita. Patients with mega-aorta syndrome would have most of the benefits from the University Penn type III procedures. We did extended replacement from the ascending aorta, arch, and descending aorta via a left thoracotomy in 22 patients with 1 hospital mortality, but this is a big operation. You have done 8 type III procedures, and I would like to know the mortality and morbidity in this specific patient group.

Dr Milewski. Of those patients, there were only 2 mortalities, and both of those patients died of multiorgan system failure.

Dr Okita. During follow-up, how many deaths did you see in the hybrid group? By looking at the Kaplan-Meier curve, although statistically insignificant, all deaths seem to occur within 1 year after surgery in the hybrid group. What was the cause of death, and are there any aorta-related events?

Dr Milewski. We are actually updating our data bank so that we have a more sophisticated ability to know the exact cause of death of the patients. We recently had a patient who was sent home to an intermediate care facility and had a tracheostomy at that point. She died in the outside facility. But at this point I cannot tell you specif- ically what each one of these patients died of; however, I can tell you that we are working on that data bank to update it.

Dr Okita. Okay. The final question is regarding indications for a hybrid procedure. In the text you gave me before, you stated that indications of total arch replacement are younger patients, collagen tissue disease (including Marfan), patients who require multiple concomitant cardiac procedures, and infection. What about indications for the hybrid procedure?

Dr Milewski. At this point that is basically what this study at the University of Pennsylvania is all about, to determine exactly what criteria to use, and it is more or less criteria building in progress. We have only been doing the procedure since 2005 , and we do not at this point have definitive criteria for our patients, but we hope this study and its continuance will provide the criteria.

Dr Joseph Bavaria (Philadelphia, $\mathrm{Pa}$ ). Because I am the one who selects the patients, I will answer that with a bit more detail. When we chose the control group, it was a combination of proximal descending aortic aneurysm repair and arch repair. The hybrid arch operation is not an operation for a standard issue proximal thoracic aortic disease process. So the selection process is basically someone who would require a very deep median sternotomy or a clamshell or something of that nature. So the selection consists of a significant arch, even a distal arch operation. That is the whole concept behind this procedure, really, that is, how to select cases.

In 2005 the Gore graft became available, so we decided to try the hybrid arch procedure to see if it would work in these particular high-risk patients who would have had an open operation before, although high risk. We performed the operation in 27 patients. We have the data: My plan is that we are going to basically perform a standard operation in young patients and a hybrid operation in older patients, as long as they have the appropriate anatomy for this procedure. 